Article

\title{
The Association between Compassionate Love and Spiritual Coping with Trauma in Men and Women Living with HIV
}

\author{
Heidemarie Kremer*, Gail Ironson, Nicoleta de Deugd and Monica Mangra \\ Department of Psychology, University of Miami, Rhodes House, Building 37, 1204 Dickinson Drive, \\ Coral Gables, FL 33146, USA; E-Mails: gironson@aol.com (G.I.); n.dedeugd@umiami.edu (N.D.); \\ mmangra92@gmail.com (M.M.)
}

* Author to whom correspondence should be addressed; E-Mail: heidemariekremer@yahoo.de; Tel.: +1-786-458-5560.

External Editor: Arndt Büssing

Received: 13 July 2014; in revised form: 22 September 2014 / Accepted: 24 September 2014 /

Published: 21 October 2014

\begin{abstract}
Our ten-year study examined the association between compassionate love (CL) - other-centered love, as well as compassionate self-love, and spiritual coping (SC) the use of spirituality (connection to a Higher Presence or God) as a means to cope with trauma, and gender differences in 177 people living with HIV (PLWH). In a secondary data analysis of six-monthly interviews/essays, we coded five criteria of CL and rated the benefit of CL giving, receiving and self for the recipient. Synergistically, we rated longitudinal SC based on coding of 18 coping strategies. Overall, mean CL towards self was very high, followed by $\mathrm{CL}$ receiving and giving, while mean $\mathrm{SC}$ was moderately high. Women, in comparison to men, perceived higher benefit from SC and giving CL to others. Overall, CL towards self had the strongest association with $\mathrm{SC}$, more pronounced in women than in men. Beyond gender, only CL for the self was a significant predictor of SC. Although there was a moderate association between SC and the perceived benefit from giving CL, after controlling for gender, this association was present in men only. Conversely, receiving CL from others yields a stronger association with $\mathrm{SC}$ in women than in men. Women perceived to benefit significantly more from SC and giving CL to others compared to men, whereas no gender differences were found on perceiving benefit from receiving CL from others or oneself. In conclusion, although women perceive more benefit from giving CL to others than men, this does not explain the higher benefit from SC among women. Ultimately, both men and women perceive to benefit more from SC the more they
\end{abstract}


exhibit CL towards self and thus spiritual counseling should keep the importance of the balance between CL towards self and others in mind.

Keywords: HIV; compassionate love; spirituality; coping; gender differences; health; behavior; counseling

\section{Introduction}

Although used since the seventeenth century [1], the expression "compassionate love" (CL) had recently emerged as the best "compromise phrase" in multicultural collaborative work conducted by the World Health Organization (WHO) in search for a measure of quality of life that includes spirituality [2]. Underwood et al. defined CL as "love that centers on the good of the other", which includes five criteria: free choice, cognitive understanding, valuing/empowering, openness, receptivity for spirituality, and response of the heart [3]. They also emphasize the dynamic process of CL: in order to give, one has to first be able to express CL towards self [4]. CL and spirituality/religion are oftentimes practiced together, as spiritual leaders and the exceptional individuals that are depicted in religious textbooks, emphasize the importance of unselfish attitude and love towards each other.

As a broad concept, spirituality can be defined as a connection to a Higher Presence (e.g., within oneself, others, nature or the transcendent) [5], whereas religiousness refers to spirituality within a religious institution. People Living with HIV (PLWH) often turn to spirituality to cope with the multiple traumas they encounter in their lives, such as death of a loved one, stigma, poverty, lack or limited access to health care [5]. To better describe longitudinal spiritual coping (SC), we developed the functional components model of coping [6] based on the transactional model of stress and coping of Lazarus and Folkman, which defines coping as a reduction of the discrepancy between the primary appraisal of the stressor and the secondary appraisal of one's ability to cope [7]. The model of Lazarus and Folkman is a precursor of ours, which shows that the reaction to the stressor should be considered as a third appraisal. Thus, an effective way of coping involves continuous formative evaluation of the three functional components: the stressor, the self and the reaction. In other words, looking back, analyzing and adjusting strategies may not show significant results if studied cross-sectional. In order to understand the dynamics of the three functional components, coping needs to be viewed in a longitudinal context [5]. For example, one who displays aversion towards God for his/her HIV diagnosis (the initial reaction to the stressor), may come overtime to the understanding that spirituality is what keeps him/her going in the face of HIV, and so post-traumatic spiritual transformation may flourish. Hence, $\mathrm{SC}$ is a dynamic process that involves feedback loops, and can best be examined in a longitudinal design.

Based on qualitative content analysis of interviews and essays of 177 people living with HIV, whom we followed longitudinally for up to ten years ${ }^{1}$, we developed a structural framework of "Compassionate Love and Health in People with HIV" based on Underwood's five criteria of giving

\footnotetext{
1 Further details in the methods section.
} 
and receiving other-centered CL [4]. During inductive coding of the first cases, we added the concept of CL towards self to our structural framework. CL is based on motivation and discernment, and the motivation of giving has to be independent of the expectancy of receiving CL. Therefore, the other-centered motivation and discernment behind any expression of CL should be first considered and thought upon. For example, making a donation to a charity in order to become famous would not be considered as an act of other-centered CL. Discernment involves the balance between self-centered and other-centered CL, such as in an airplane emergency putting on one's oxygen mask first before helping others. Altruism and other-centeredness can go as far as self-sacrifice and by not being mindful of one's own capacity to give, one can go as far as self-injuring.

The word "altruism" (from Latin alter: "other") was coined by the French founder of positivism, Auguste Comte, in order to describe the ethical doctrine he supported [8]. He believed that individuals had a moral obligation to renounce self-interest and live for others. However, in everyday life people tend to think of altruism from an economic standpoint, and most studies on this matter refer mostly to the financial aspect of an altruistic act. In a study on altruism and gender differences [9], it was found that women are kinder when altruism is expensive, but when it's cheap, men tend to be more altruistic. Another finding on the effects of gender and CL [10], shows that women would expect to experience enhanced positive mood as a result of both giving and receiving help in a relational context. Moreover, CL towards others was shown to help enhance the positive mood from giving and receiving.

In our previous study on CL, we found that high CL towards self had the strongest association with high CL receiving [4]. Participants with high CL towards self were also more likely to give CL to others. In contrast, our study showed that participants with low CL towards self were less likely to receive and give CL. Furthermore, high CL towards self had the strongest association with high CL receiving $(p<0.001)$, and was related to expressing high CL towards others $(p=0.001)$. In summary, CL towards self and others was reciprocal, as well as it was the intensity of giving and receiving CLthe more one gives CL, the more CL one receives. CL self was almost congruent with the full expression of CL, and this was shown by the correlation between CL self and the sum of CL (self, giving, and receiving).

We also coded the frequency of Underwood's five criteria of CL with respect to its three components: giving, receiving and the self. As described in our previous study on CL [4], the most frequent criterion coded was free choice, followed by cognitive understanding, valuing/empowering, response of the heart and openness/receptivity for spirituality.

According to the adjusted $\mathrm{R}^{2}$, the five criteria explained $53 \%$ of variance in CL giving (33\% valuing/empowering, $10 \%$ cognitive understanding, $10 \%$ other three criteria), $40 \%$ of variance in $\mathrm{CL}$ receiving (19\% valuing/empowering, $11 \%$ free choice, $10 \%$ other three criteria), and $48 \%$ of variance in CL self (29\% valuing/empowering, 10\% cognitive understanding, 9\% other three criteria).

Based on the same interviews/essays, we also examined strategies of SC and developed a rating scale for longitudinal SC with trauma [5]. Our study found that most participants were spiritual and used spiritual practices, while the connection to a Higher Presence was found whether via prayer or meditation. Although spiritual comfort, empowerment, growth/transformation, and gratitude were frequent positive aspects of spirituality, spirituality was less involved in the many ways of meaning-making, positive reframing, and community support. Surrender was a double-edged sword, since the belief that "God is in control" could result in both overcoming substance use and not taking HIV medication. 
A critical confrontation with spirituality, illustrated by less common aspects, such as conflict, struggle, guilt, anger, and disengagement, was found to possibly catalyze both a spiral of negative effects and a positive transformation via an increase in spirituality.

We found that $65 \%$ used spirituality to cope in a positive way (as a source of comfort, empowerment, growth/transformation, gratitude, and community support), $7 \%$ used it negatively (spiritual coping had a long-lasting negative effect and became at times even a stressor in itself), and $28 \%$ made no significant use of spirituality [5]. Similar to other studies [11-13] women, heterosexuals and African-Americans made positive use of spirituality, while Non-Latino Whites used spirituality less.

Longitudinal SC was a stronger predictor of biological outcomes (CD4 cell preservation and cumulative undetectable viral load) than CL [14]. In fact, only CL towards self but not other-centered CL, was associated with biological outcome [4]. In an additional study on PLWH [15], we have shown that the spiritual component of CL can also manifest through a view of God/Higher Presence as loving/benevolent. For example, some participants felt being "selected by God" to have HIV. Such positive spiritual reframing can induce spiritual transformation and enhance practices of CL [16]. Others perceived themselves as rejected, needless, unloved and unworthy [16-18], which may have led to isolation and inability to express and feel CL [4]. A loving/benevolent view of God [15] was the strongest predictor of biological outcome, stronger than SC [5], increase in spirituality after diagnosis [19], view of God/Higher Presence as hard/punishing [15] depression, and avoidant coping [20].

Thus far, we did not examine the association between CL and longitudinal SC with trauma in our study. Therefore, the purpose of this study is to shed some light on the relationship between the three components of CL (giving, receiving and the self) and longitudinal SC with trauma, as well as to examine if there are gender differences. We hypothesize that in PLWH, CL-giving, receiving and self, and SC are associated; however those associations differ between men and women. We further hypothesize that CL predicts SC, even beyond gender.

\section{Methods}

For up to ten years, we interviewed 177 men and women living with HIV every six months and examined the psychosocial and spiritual factors related to health and longevity [15,20], using a mixed method approach. In a secondary data analysis of the interviews/essays, we applied qualitative content analysis $[21,22]$ to code the presence of the five criteria of CL (free choice, cognitive understanding, valuing/empowering, openness and receptivity for spirituality, and response of the heart) within all three components (giving, receiving, and self) and rated the benefit of CL for the recipient on a 6-point Likert scale [4]. In addition, we developed an overall rating scale of longitudinal SC based on the coding of 18 coping strategies [5].

\subsection{Study Population and Sampling}

Between 1997 and 2000, 177 PLWH were recruited via newspaper ads, word of mouth and flyers distributed through various channels: doctors' offices, community events, support groups and HIV organizations. The sample was diverse for gender (30\% female), age (mean age $37.49 \pm 8.88$ years), ethnicity (36\% African American, 31\% Non-Latino White, 28\% Latino), and sexual orientation (45\% heterosexual). Entry criteria were 150-500 CD4-cells/mm3, CD4-nadir $>75$ cells $/ \mathrm{mm}$, and with no 
prior AIDS-defining symptoms or HIV associated dementia, due to our hypothesis that psychological factors would have maximum impact at this disease stage. In order to reduce unreliability or confounders for immune response, people with active substance use disorder, and/or psychosis (based on the Structured Clinical Interview for DSM-III-R [23] were also excluded. Most participants were relatively poor, with $60 \%$ living on less than $\$ 10 \mathrm{k}$ annual income, which is consistent with their high unemployment (15\%) and disability (42\%). About half (52\%) did not attend religious services, 23\% less than weekly, and $25 \%$ at least weekly.

\subsection{Procedures}

The study was IRB approved and took place at the University of Miami. Participants gave informed consent and received \$50 compensation following every visit. We collected our data-detailed interviews and essays in six-month intervals-between 1997 and 2010. At each visit, participants completed a comprehensive interview and a questionnaire, including sociodemographics, psychological, behavioral, social, and spiritual factors related to health (see [20] for full details). Due to the cohort effect, 66/177 participants (37\%) remained in the study after four years while the rest dropped out due to loss of follow-up, death, health deterioration, relocation, and other reasons. Accounting for death $(26 / 177,15 \%)$, we had an average 270 dropout rate of $14 \%$ participants/year with $44 \%$ of those alive remaining in the sample after four years.

Between 2010 and 2012, we transcribed and performed in-depth qualitative data analysis. On the life-event scale (from -3 to +3 ) [24], participants rated the level of stress that they had experienced in the last six months. For each participant, we fully transcribed the interview of the time-point with the highest negative rating (closest to -3 , very stressful) during the first three years of study. For some participants, more than one interview was fully transcribed if it contained relevant information on SC or CL. A team of ten trained transcribers summarized the content of all other interviews and essays for up to ten years. All transcripts were quality controlled, entered in the qualitative software Atlas.ti ${ }^{\circledR}$ and analyzed using directed qualitative content analysis [21,22]. Each time a full transcript, including one or more interviews and a detailed summary of all other essays/interviews, was completed, the transcriber worked with the research team inductively line by line through the entire transcript to highlight quotes and anchor examples. The text passage quote was the smallest unit of our analysis to develop a tentative categorization system. The definitions were fine-tuned until a point of saturation was reached and a coding/rating agenda with sufficient inter-rater reliability was established. Finally, two raters analyzed all 177 transcripts independently. Each rater provided quotes for each rating of CL (giving/receiving/self) and longitudinal SC. Any discrepancies $=1$ were either consensually agreed or discussed in the team. For the rating, any discrepancies $>1$ were quality controlled by another rater-couple, again coding independently and reviewing discrepancies consensually. For further statistical analysis, the CL and SC ratings were aggregated and transferred into SPSS ${ }^{\circledR}$ version 19.

\subsection{Interviews and Essays}

Initially, we asked participants about how they think they acquired HIV, and their first reaction to their diagnosis. We also asked about disclosure of their HIV diagnosis and (if applicable) of being homosexual and how this disclosure was received by other people; about their relationship status and 
practices of safe sex, and about daily routines and what they were looking forward to. Participants were asked how they were coping with HIV and other life traumas, what enabled them to move on, and if they experienced any positive results of living with HIV. Furthermore, we asked whether they had someone who can take care of them when needed, or who provided emotional support, and if they had lost a close friend or relative to AIDS. We also recorded information regarding their medical care and their relationship to their physician. Similar to the recommendations of King and Koenig [25] for the exploration of spirituality in the clinical setting, we asked our participants about their spiritual and religious beliefs (e.g., heaven, hell, karma) and probed if those beliefs played a role in coping. They also wrote essays about how they coped with the most difficult event that had happened to them in the last six months.

\subsection{Rating of Compassionate Love}

Inductively, we searched for additional components of CL and a method to rate the benefit of CL for the recipient. After analyzing 20 transcripts, inductive coding revealed that CL towards self was an additional component that should be included in the ratings (see introduction). We coded the presence of the five criteria for each component of CL, giving, receiving and self, grounded in at least one characteristic quotation. Further, we developed a 6-point Likert scale rating for the benefit of CL for the recipient (ranging from $1=\mathrm{CL}$ negative or harmful to $6=\mathrm{CL}$ is a central element in the person's life), which was based on the overall coding of the transcript.

We compared participants with low scores on CL, which denotes (1) negative or harmful CL; (2) no expression of CL; or (3) attempted but not beneficial CL, with participants with high scores who (4) perceived CL as beneficial but not empowering; (5) beneficial and empowering, and (6) as a central element in the person's life (6).

The results of this analysis are summarized in our introduction and our original article [4]. The finetuned definitions of the codes of the five criteria had substantial reliability (Cohen's Kappa above 0.60) and reliability of the rating of the benefit of CL was excellent (Kendall's tau $\mathrm{B}=0.81, p<0.001$ ) [26]. In case there was not enough information for a reliable rating, we excluded the case from further analysis. Not enough information applied to 20 ratings of the benefit of CL (involving $14 \mathrm{CL}$ giving, $2 \mathrm{CL}$ receiving, and 4 CL self).

\subsection{Rating of Spiritual Coping}

For longitudinal SC a point of saturation was reached after more than 70 interviews. Analyzing 20 interviews, ten raters established independent inter-rater reliability. For each Cronbach's alpha $<0.80$, definitions were revised. Next, chance-corrected inter-rater reliability of two raters was established for 20 further interviews. The definitions were fine-tuned until reliability of the coding was substantial (Cohen's Kappa above 0.60) and reliability of the overall SC rating was excellent (Kendall's tau $\mathrm{B}=0.81, p<0.001$ ). In one case, there was not enough information to rate overall longitudinal SC reliably. The 9-point scale rated participants from -4 (most stressful) to +4 (spiritual transformation). For instance, a continuous spiritual seeker who feels lost in life and attempts suicide is categorized as -4 , however a spiritual experience triggering a positive transformation is rated as +4 . Persistent struggle with unquestioned spiritual beliefs (e.g., karma, hell) (-3) is opposed to post-traumatic spiritual 
growth/transformation (+3). Ambivalent SC with fluctuating effects is categorized as -2 , whereas finding benefit in consistent spiritual practices is categorized as +2 . The absence of elaborate use or any effect of SC was rated as +1 and feeling guilty about not having a spiritual connection as -1 [5].

\subsection{Statistical Analysis}

Descriptive statistics were calculated for the ratings of CL (giving, receiving, self) and longitudinal $\mathrm{SC}$ for men and women. Independent Student's t-tests calculated gender differences. To examine the relationship between CL and SC, we calculated the overall Pearson's correlations, partial Pearson's correlations (controlling for gender), as well as the Pearson's correlations in women only and men only.

In addition, we ran multiple linear regression analyses, first using CL (giving, receiving, self) as predictors of SC and a second model entering gender (block 1) and CL giving, receiving and self (block 2) as predictors of SC.

\section{Results}

After presenting the descriptive statistics of CL giving, receiving and self, and longitudinal SC by gender, we test our hypothesis of the relationship between CL and SC and the gender difference of this relationship.

\subsection{Frequencies of CL and SC by Gender}

Table 1 shows the mean differences between men and women on the CL rating and the longitudinal $\mathrm{SC}$ rating. Overall, mean CL towards self was very high, closely followed by CL receiving and giving, while mean SC was moderately high. Independent Student's t-tests showed that women compared to men perceived to benefit significantly more from SC and giving CL to others, whereas no gender differences were found on perceiving benefit from receiving CL from others or from CL towards self.

Table 1. Differences between men and women on compassionate love (CL) and spiritual coping (SC).

\begin{tabular}{lcccc}
\hline & $\begin{array}{c}\text { Men }(\mathbf{n}=\mathbf{1 2 4}) \\
\mathbf{M} \pm \mathbf{S D}\end{array}$ & $\begin{array}{c}\text { Women }(\mathbf{n}=\mathbf{5 3}) \\
\mathbf{M} \pm \mathbf{S D}\end{array}$ & $\begin{array}{c}\text { Total }(\mathbf{N}=\mathbf{1 7 7}) \\
\mathbf{M} \pm \mathbf{S D}\end{array}$ & \multirow{2}{*}{$\boldsymbol{*}^{*}$} \\
\hline CL Giving & $3.82 \pm 1.24$ & $4.31 \pm 1.12$ & $3.97 \pm 1.22$ & 0.020 \\
CL Receiving & $3.98 \pm 0.92$ & $3.98 \pm 1.02$ & $3.98 \pm 0.95$ & 0.985 \\
CL Self & $4.18 \pm 1.07$ & $4.00 \pm 1.17$ & $4.13 \pm 1.10$ & 0.326 \\
SC & $1.47 \pm 1.76$ & $2.33 \pm 1.41$ & $1.72 \pm 1.71$ & 0.002 \\
\hline
\end{tabular}

Note: * two-tailed test.

\subsection{Association between CL and SC Accounting for Gender}

In line with our hypothesis, we found an association between the three components of CL and longitudinal SC, as shown in Table 2. SC was strongest associated with CL towards self and this link was more pronounced in women than in men. SC Overall was stronger associated with giving than with receiving CL. However, after controlling for gender, this difference flipped. In women, receiving $\mathrm{CL}$ from others was strongly associated with $\mathrm{SC}$, but in men this association was only weak. A 
moderate association between SC and the experience of benefits from giving CL to others was only present in men but not in women.

Table 2. Pearson's correlations between spiritual coping (SC) and compassionate love (CL): overall, partial ${ }^{\mathrm{a}}$, women only, and men only.

\begin{tabular}{lccc}
\hline & CL giving & CL receiving & CL self \\
\hline SC Overall & $0.28 * *$ & $0.25 * *$ & $0.34 * * *$ \\
SC Partial ${ }^{\text {a }}$ & $0.25 * *$ & $0.27 * *$ & $0.38 * * *$ \\
SC Women only & 0.23 & $0.41 * *$ & $0.45 * *$ \\
SC Men only & $0.26 * *$ & $0.21 * *$ & $0.35 * * *$ \\
\hline \multicolumn{2}{r}{ Notes: $^{\text {a }}$ controlling for gender; $* p<0.05 ; * * p<0.001 ; * * * p<0.0001}$.
\end{tabular}

\subsection{CL as a Predictor of SC beyond Gender}

The first multiple linear regression analysis revealed that CL giving, receiving, and self together explained $15 \%$ of variance in $\mathrm{SC}\left(\mathrm{F}_{3153}=8.97, p<0.001\right)$. Only $\mathrm{CL}$ for the self was a significant predictor (beta $=0.27, S E=0.16, p=0.007$ ) but CL giving trended to predict better SC (beta $=0.16$, $S E=0.13, p=0.068)$. The second regression model showed that gender alone accounted for $5 \%$ of variance in $\mathrm{SC}\left(\mathrm{F}_{1155}=8.70, p<0.004\right)$ and gender together with $\mathrm{CL}$ giving, receiving, and self-account for $20 \%$ of variance $\left(\mathrm{F}_{1155}=8.70, p<0.004\right)$. In the second model, gender $($ beta $=0.23$, $S E=0.29, p=0.003$ ) and $\mathrm{CL}$ for the self (beta $=0.31, S E=0.16, p=0.002$ ) were significant predictors of SC and there was no longer a trend for CL giving as a predictor (beta $=0.09, S E=0.13$, $p=0.003)$. Thus, $\mathrm{CL}$ for the self is a significant predictor of SC beyond gender.

\section{Discussion}

This study analyzed the relationship between longitudinal SC with trauma and the benefits of CL in men and women living with HIV. Three components of CL were analyzed, PLWH perceiving to benefit from: (1) giving other-centered CL; (2) receiving other-centered CL; and (3) CL towards self.

We found that both men and women perceived on average a very high benefit from all components of CL, whereas averages of benefit from SC were moderately high. Women compared to men, perceived more benefit from SC and giving CL to others. Beyond gender, only CL for the self-remained a significant predictor of SC.

The results of our study dovetail with other findings that highlight women's importance of spirituality for their health and well-being [27]. Similar to other studies, where most women reported that support from others, prayer, and trusting in God played some role in their ability to cope with their HIV/AIDS status [28], our finding shows that for women receiving CL from others was strongly associated with SC. On a general note, CL in both men and women was found to be positively associated with prosocial behavior towards close others and all of humanity [10].

Our hypothesis on an association between CL and SC was confirmed for all components of CL, mostly for CL towards self, which, according to our prior study [4], is the seed of other-centered CL.

What this study adds to the existing literature, is that for women SC is more beneficial the more they feel being compassionately loved by others or by themselves. Despite that women, in comparison 
to men, have a greater tendency to turn to spirituality for coping and altruistic other-centered love, we did not find a significant connection between their personal benefit from using spirituality to cope and providing CL towards others.

\section{Conclusions}

Although the results of this study are merely correlational and do not allow any causal conclusions, these gender differences may have applications for spiritual counseling. As pointed out in the introduction, the full expression of CL hinges on the balance between self- and other-centered CL. Ultimately, both men and women perceive to benefit more from SC the more they exhibit CL towards self and thus spiritual counseling should keep the importance of the balance between CL towards self and others in mind. As far as giving and receiving other-centered CL is concerned, spiritual counseling may have to look closer on women's perceptions of the CL they receive from others rather than the CL they give to others. In women, it is unclear if their perceived benefit from giving CL to others is helpful for their own personal SC with HIV. Since SC is a stronger predictor of biological outcome than CL $[4,14]$, future research should examine why women compared to men living with HIV seem to benefit more from SC, taking sexual orientation, ethnicity, socioeconomic status and geographical background into account [5].

In conclusion, CL towards self is associated with better SC in both men and women living with HIV.

\section{Acknowledgements}

We thank the John Templeton Foundation and the Fetzer Institute for the funding of this secondary data analysis, and the NIH (R01MH53791 and R01MH066697, PI: Gail Ironson) and the Metanexus Foundation for financing the longitudinal study. Further, we thank all people living with HIV for sharing their personal experiences with us. We thank the positive survivors research team for running the longitudinal study, in particular Annie George for conducting most of the interviews, the research assistants Tony Guerra and Marietta Suarez for their help with the coding agenda, Franz Lutz for his suggestion to create a Wiki for the coding agenda, and all the students for their enormous help in transcribing and coding of the interviews and essays. In addition, we thank Wayne Ramsey for extending the grant from the Fetzer Institute, which made this manuscript possible.

\section{Author Contributions}

HK conceived the secondary analysis, designed and developed the qualitative content analysis, performed statistical analysis and drafted the manuscript together with NDD. GI is the PI of the main study and designed the interview, assisted in the qualitative analysis and helped to draft the manuscript together with MM. All authors read and approved the final manuscript.

\section{Abbreviations}

CL: compassionate love;

SC: spiritual coping;

HIV: human immunodeficiency virus; 
PLWH: people living with HIV;

WHO: World Health Organization.

\section{Conflicts of Interest}

The authors declare no conflict of interest.

\section{References}

1. Law, William. "A Collection of Letters on the most Interesting and Important Subjects, and on several Occasions." Available online: http://www.ccel.org/l/law/letters/let.htm (accessed on 26 September 2014).

2. Underwood, Lynn G. "Compassionate Love: A Framework for Research." In The Science of Compassionate Love: Theory, Research, and Applications. Edited by Beverley Fehr, Susan Sprecher and Lynn G. Underwood. Hoboken: Wiley-Blackwell, 2008, vol. 1.

3. Fetzer Institute. "Compassionate Love in a Relational Context." 2012. Available online: http://www.fetzer.org/resources/compassionate-love-relational-context (accessed on 26 September 2014).

4. Kremer, Heidemarie, Gail Ironson, Lauren Kaplan, Rick Stuetzle, and Mary A. Fletcher. "Compassionate Love as a Predictor of Reduced HIV Disease Progression and Transmission Risk." Evidence-Based Complementary and Alternative Medicine 2013 (2013): 1-13. doi:10.1155/2013/819021.

5. Kremer, Heidemarie, and Gail Ironson. "Longitudinal Spiritual Coping with Trauma in People with HIV: Implications for Health Care.” AIDS Patient Care and STDs 28 (2014): 144-54.

6. Ironson, Gail, and Heidemarie Kremer. "Coping, Spirituality, and Health in HIV." In The Oxford Handbook of Stress, Health, and Coping, 1st ed. Edited by Susan Folkman. Oxford, UK: Oxford University Press, 2011.

7. Lazarus, Richard S., and Susan Folkman. Stress, Appraisal, and Coping. New York: Springer, 1984.

8. Comte, Auguste. Catechism of Positivism. Translated by Richard Congreve. Edited by Paul Kegan. Cambridge: Cambridge University Press, 2009.

9. Andreoni, James, and Lise Vesterlund. "Which is the Fair Sex? Gender Differences in Altruism." The Quarterly Journal of Economics 116 (2001): 293-312.

10. Sprecher, Susan, Beverley Fehr, and Corinne Zimmerman. "Expectation for Mood Enhancement as a Result of Helping: The Effects of Gender and Compassionate Love." Sex Roles 56 (2007): 543-49.

11. Coleman, Christopher L., William L. Holzemer, Lucille S. Eller, Inge Corless, Nancy Reynolds, Kathleen M. Nokes, Jeanne K. Kemppainen, Pam Dole, Kenn Kirksey, Liz Seficik, et al. "Gender Differences in Use of Prayer as a Self-Care Strategy for Managing Symptoms in African Americans Living with HIV/AIDS." The Journal of the Association of Nurses in AIDS Care 17 (2006): 16-23.

12. Cotton, Sian, Christina M. Puchalski, Susan N. Sherman, Joseph M. Mrus, Amy H. Peterman, Judith Feinberg, Kenneth I. Pargament, Amy C. Justice, Anthony C. Leonard, and Joel Tsevat. 
"Spirituality and Religion in Patients with HIV/AIDS." Journal of General Internal Medicine 21 (2006): S5-13.

13. McIntosh, Roger C., and Monics Rosselli. "Stress and Coping in Women Living with HIV: A Meta-Analytic Review." AIDS and Behavior 16 (2012): 2144-59.

14. Kremer, Heidemarie, Gail Ironson, Lauren Kaplan, Rick Stuetzele, Neil Baker, and Mary Ann Fletcher. "Spiritual Coping Predicts CD4-Cell Preservation and Undetectable Viral Load Over Four Years." AIDS Care, 2014, doi:10.1080/09540121.2014.952220. Available online: http://www.tandfonline.com/doi/abs/10.1080/09540121.2014.952220\#.VEAiBvnF9AB (accessed on 16 October 2014).

15. Ironson, Gail, Rick Stuetzle, Dale Ironson, Elizabeth Balbin, Heidemarie Kremer, Annie George, Neil Schneiderman, and Mary A. Fletcher. "View of God as Benevolent and Forgiving or Punishing and Judgmental Predicts HIV Disease Progression.” Journal of Behavioral Medicine 34 (2011): 414-25.

16. Kremer, Heidemarie, Gail Ironson, and Lauren Kaplan. "The Fork in the Road: HIV as a Potential Positive Turning Point and the Role of Spirituality.” AIDS Care 21 (2009): 368-77.

17. Ironson, Gail, Heidemarie Kremer, and Dale Ironson. "Spirituality, Spiritual Experiences, and Spiritual Transformations in the Face of HIV." In Spiritual Transformation and Healing: Anthropological, Theological, Neuroscientific, and Clinical Perspectives, 1st ed. Edited by Joan D. Koss-Chioino and Philip Hefner. Lanham: AltaMira Press, 2006, pp. 241-62.

18. Kremer, Heidemarie, and Gail Ironson. "Everything Changed: Spiritual Transformation in People with HIV." International Journal of Psychiatry in Medicine 32 (2009): 243-62.

19. Ironson, Gail, Rick Stuetzle, and Mary A. Fletcher. "An Increase in Religiousness/Spirituality Occurs after HIV Diagnosis and Predicts Slower Disease Progression over 4 Years in People with HIV.” Journal of General Internal Medicine 21 (2006): S62-68.

20. Ironson, Gail, Conall O’Cleirigh, Mary A. Fletcher, Jean Phillippe Laurenceau, Elizabeth Balbin, Nancy Klimas, Neil Schneiderman, and George Solomon. "Psychosocial Factors Predict CD4 and Viral Load Change in Men and Women with Human Immunodeficiency Virus in the Era of Highly Active Antiretroviral Treatment.” Psychosomatic Medicine 67 (2005): 1013-21.

21. Mayring, Philipp. "Qualitative Content Analysis.” Forum: Qualitative Social Research 1 (2000): Art. 20. Available online: http://nbn-resolving.de/urn:nbn:de:0114-fqs0002204 (accessed on 26 September 2014).

22. Hsieh, Hsiu-Fang, and Sarah E. Shannon. "Three Approaches to Qualitative Content Analysis." Qualitative Health Research 15 (2005): 1277-88.

23. Spitzer, Robert L., Janet B. W. Williams, Miriam Gibbon, and Michael B. First. "The Structured Clinical Interview for DSM-III-R (SCID) I: History, Rationale, and Description." Archives of General Psychiatry 49 (1992): 624-29.

24. Sarason, Irwin G., James H. Johnson, and Judith M. Siegel. "Assessing the Impact of Life Changes: Development of the Life Experiences Survey." Journal of Consulting and Clinical Psychology 46 (1978): 932-46.

25. King, Michael B., and Harold G. Koenig. "Conceptualising Spirituality for Medical Research and Health Service Provision." BMC Health Services Research 9 (2009): 116. Available online: http://www.biomedcentral.com/1472-6963/9/116 (accessed on 26 September 2014). 
26. Landis, J. Richard, and Gary G. Koch. "The Measurement of Observer Agreement for Categorical Data." Biometrics 33 (1977): 159-74.

27. Dalmida, Safiya George, Marcia Mcdonnell Holstad, Colleen DiIorio, and Gary Laderman. "The meaning and use of spirituality among African American women living with HIV/AIDS." Western Journal of Nursing Research 34 (2012): 736-65.

28. Hodge, David R., and Jini L. Roby. "Sub-Saharan African Women Living with HIV/AIDS: An Exploration of General and Spiritual Coping Strategies.” Social Work 55 (2010): 27-37.

(C) 2014 by the authors; licensee MDPI, Basel, Switzerland. This article is an open access article distributed under the terms and conditions of the Creative Commons Attribution license (http://creativecommons.org/licenses/by/4.0/). 